\title{
CONTINUOUS AND DISCRETE FRAMES GENERATED BY THE EVOLUTION FLOW OF THE SCHRÖDINGER EQUATION
}

\author{
GIOVANNI S. ALBERTI, STEPHAN DAHLKE, FILIPPO DE MARI, ERNESTO DE VITO, \\ AND STEFANO VIGOGNA
}

\begin{abstract}
We study a family of coherent states, called Schrödingerlets, both in the continuous and discrete setting. They are defined in terms of the Schrödinger equation of a free quantum particle and some of its invariant transformations.
\end{abstract}

\section{INTRODUCTION}

In Quantum Mechanics, the time evolution of a $d$-dimensional free particle is described by the Schrödinger equation

$$
\left\{\begin{array}{l}
i \frac{\partial}{\partial t} f(x, t)=-\frac{1}{2 \pi} \Delta f(x, t) \\
f(\cdot, 0)=f_{0},
\end{array}\right.
$$

where $\Delta$ is the Laplace operator acting on the "space" variable $x \in \mathbb{R}^{d}$, and $f_{0}$ is a square-integrable function on $\mathbb{R}^{d}$ describing the state of the quantum particle at time zero (for the sake of simplicity, the mass is normalized so that the Laplacian has the simple factor $1 / 2 \pi)$.

The aim of this paper is to introduce a new family of coherent states (i.e. a frame) generated by the time evolution unitary operator defined by the Schrödinger equation; following [1, 2, 3, its elements are called Schrödingerlets.

Clearly, the time evolution operator $e^{i \frac{t}{2 \pi} \Delta}$ is not enough to generate a frame for $L^{2}\left(\mathbb{R}^{d}\right)$, hence we need to add other unitary transformations. Observe that equation (1.1) is invariant both with respect to the rotations $R \in \mathrm{SO}(d)$, under the canonical action

$$
f(x, t) \mapsto f(R x, t),
$$

and with respect to the dilations $a \in \mathbb{R}_{+}$, under the parabolic action

$$
f(x, t) \mapsto a^{\frac{d}{4}} f(\sqrt{a} x, a t),
$$

where the factor $a^{\frac{d}{4}}$ ensures that the $L^{2}$-norm of $f(\cdot, t)$ is preserved. Thus, it is natural to consider the group $G=\left(\mathbb{R} \rtimes \mathbb{R}_{+}\right) \times \mathrm{SO}(d)$, i.e. the direct product of the identity component of the one-dimensional affine group and $\mathrm{SO}(d)$, and the corresponding unitary representation $\pi$ acting on $L^{2}\left(\mathbb{R}^{d}\right)$ as

$$
\pi(t, a, R) f=a^{-\frac{d}{4}} e^{i \frac{t}{2 \pi} \Delta} f_{a, R},
$$

Date: November 3, 2016.

2010 Mathematics Subject Classification. 22D10, 42C40, 42C15.

Key words and phrases. Schrödinger equation; unitary representations; frames; wavelets; coherent states; reproducing formulae; Schrödingerlets. 
where $f_{a, R}(x)=f\left(a^{-\frac{1}{2}} R^{-1} x\right)$. It follows that the solution of (1.1) is given by

$$
f(x, t)=\pi(t, 1, \mathrm{I}) f_{0}(x),
$$

and for any rotation $R \in \mathrm{SO}(d)$

$$
f(R x, t)=\pi\left(t, 1, R^{-1}\right) f_{0}(x),
$$

whereas for any dilation $a \in \mathbb{R}_{+}$

$$
a^{\frac{d}{4}} f(\sqrt{a} x, a t)=\pi\left(t, a^{-1}, \mathrm{I}\right) f_{0}(x) .
$$

Our goal is to study the properties of the corresponding family of coherent states $\{\pi(x) \eta\}_{x \in G}$ where $\eta$ is a suitable "ground state", i.e. an admissible vector. In the context of signal analysis, this amounts to analyzing the voice transform

$$
f \mapsto\langle f, \pi(\cdot) \eta\rangle
$$

as a map from $L^{2}\left(\mathbb{R}^{d}\right)$ into a suitable Hilbert space of functions on $G$. We restrict ourselves to the $L^{2}$-framework, both in the continuous and in the discrete setting. Our main contribution is twofold. First, we show that $\pi$ is a reproducing representation of $G$ and we characterize its admissible vectors. This result was already known for $d=2$ [3], and here we extend the proof to arbitrary $d$. Furthermore, we construct a discrete Parseval frame of the form $\left\{\pi\left(x_{i}\right) \eta\right\}_{i \in I}$, where $\left\{x_{i}\right\}_{i \in I}$ is a suitable sampling of $G$.

In Section 2 we introduce the Schrödingerlets in two dimensions and we discuss the construction of a Parseval frame of two-dimensional Schrödingerlets. The purpose of this dimensionality restriction is twofold. Firstly, it allows to present the main ideas of this work in a simpler way, so that it may serve as a good introduction to the more involved general setting. Secondly, the two-dimensional case is somehow different from the higher dimensional cases, since when $d=2$ the spherical harmonics on $S^{d-1}$ correspond to the standard Fourier series; thus, a separate presentation allows to underline the peculiarities of the case $d=2$.

Section 3 is devoted to studying the Schrödingerlets in any dimension. Proposition 3.3 shows that $\pi$ is a reproducing representation and characterizes its admissible vectors. As a consequence, the Schrödingerlet voice transform permits to represent the quantum states as continuous functions on the parameter space $\mathbb{R} \times \mathbb{R}_{+} \times \mathrm{SO}(d)$. Time evolution and rotations correspond to translations in the first and third variable, respectively, whereas dilations give rise to a multi-scale analysis of the original quantum state. However, the usual interpretation of multidimensional wavelets as a combination of (multi-dimensional) translations and dilations fails to apply here: translations are only one-dimensional, and act on the radial variable in the frequency domain, as we shall see below. To give a physical intuition, let us consider the $3 D$ case where $f$, up to a normalization, can be regarded as the state of a quantum spinless particle and $\eta$ as a probe state. If the momentum distribution of $\eta$ is concentrated around a ball with center $p_{0} \neq 0$ and radius $\delta$, clearly the momentum distribution of $\psi=\pi(0, a, R) \eta$ is concentrated around a ball with center in $p=R p_{0} / a$ and radius $\delta / a$. Hence $\psi(t)=\pi(t) \psi$ is the free quantum evolution of the state $\psi$ and the probe particle moves in the direction of $p$. It follows that $|\langle f, \pi(t, a, R) \eta\rangle|^{2}$ is the transition probability between $f$ and $\psi(t)$, where $a$ controls the scale change, $R$ the rotation and $t$ the time. For the classical directional wavelet transform, $a$ and $R$ play the same role, but the quantum evolution of $\psi$ is replaced by a $3 D$ translation in the space. We note that the 
lack of spatial translations in Schoredingerlets makes hard (if not impossible) to use them for a micro-local analysis, as for example wavefront sets resolution of the signals.

The main result of the paper is Theorem 3.4, which provides sufficient conditions in order to have a Parseval discrete frame.

We refer to [4, 5] for a general introduction to coherent states and reproducing formulæ associated with unitary representations. Schrödingerlets in dimension two were first introduced in [3] and further discussed in [1, 2], where $G$ is regarded as a closed subgroup of the symplectic group and $\pi$ is equivalent to the restriction to $G$ of the metaplectic representation, whose role in signal analysis has been investigated in a series of papers [6, 7, 8, 9, 10. We remark that the representation $\pi$ is reducible and its reproducing kernel is not integrable. Hence, we cannot directly apply the classical theory of square-integrable representations by Duflo and Moore [11, nor the coorbit space theory developed by Feichtinger and Gröchenig [12, 13].

Another construction of reproducing representations based on the covariance properties of a free quantum particle is given by the coherent states associated to the isochronous Galilei group (see [4, Chapter 8.4.2] and references therein). However, in this case, the dilations are not present and the frame does not depend on the time parameter. Indeed, in order to make the representation square-integrable it is necessary to reduce the Galilei group by taking the quotient modulo a group that contains the time translations.

The proof that $\pi$ is a reproducing representation is based on the general theory developed in 3 . However, in our case, the fact that $\pi$ is the direct sum of a countable family of square-integrable representations $\pi_{i}$ causes additional difficulties. A general approach to obtain a discrete frame without assuming that the kernel is in $L^{1}(G)$ has been developed in [14, 15, 16, 17, but it requires the boundedness of a suitable convolution operator (see condition (R3) of [14]), which is hard to prove in our setting. We follow here a different approach. Taking into account that $\pi=\bigoplus_{i} \pi_{i}$, the discretization is achieved by a slight generalization of a well known result on discrete wavelet frames in $L^{2}(\mathbb{R})$ [18, Theorem 1.6, Chapter 7], by Schur's orthogonality relations for finite groups and a technical lemma about Parseval frames (Lemma 3.6). Comparing with the approach taken in 14, we are able to provide only Hilbert frames; we hope to extend our results in future work and succeed in describing Banach frames related to the function spaces introduced in 15, 16, 19. We also plan to investigate the relation between the decay of the Schrödingerlet coefficients and the smoothness properties of the analyzed function.

\section{THE MAIN RESULT IN TWO DIMENSIONS}

We state here the main result of this paper particularized for two-dimensional signals. In the first part of the section we introduce the continuous Schrödingerlets following [19].

2.1. The continuous Schrödingerlets in $\mathbf{2 D}$. For $d=2$ we identify the abelian group $\mathrm{SO}(2)$ with the one dimensional torus $\mathcal{T}=\mathbb{R} / 2 \pi \mathbb{Z}$ as

$$
\theta \longleftrightarrow R_{\theta}=\left[\begin{array}{cc}
\cos \theta & -\sin \theta \\
\sin \theta & \cos \theta
\end{array}\right]
$$

The group $G$ is $\left(\mathbb{R} \rtimes \mathbb{R}_{+}\right) \times \mathcal{T}$ and its elements are denoted by $(b, a, \theta)$, writing $b$ instead of the time variable $t$. In order to better visualize the action of $\pi$ given 
by (1.2), it is worth rewriting it in an equivalent formulation by means of an intertwining operator $S$ which we shall now define. We work in the Fourier domain with polar coordinates, and then perform a Fourier series with respect to the angular variable.

Below we write $\widehat{\mathbb{R}}^{2}$ for the dual space to $\mathbb{R}^{2}$ and $\mathrm{d} \xi$ and $\mathrm{d} x$ for the corresponding Lebesgue measures. We denote by $\mathrm{d} \theta$ the Riemannian measure of $\mathcal{T}$ (so that $\left.\int_{\mathcal{T}} \mathrm{d} \theta=2 \pi\right)$. We let $\mathcal{F}: L^{2}\left(\mathbb{R}^{2}\right) \rightarrow L^{2}\left(\widehat{\mathbb{R}}^{2}\right)$ denote the Fourier transform given by

$$
\mathcal{F} f(\xi)=\int_{\mathbb{R}^{2}} f(x) e^{-2 \pi i x \cdot \xi} \mathrm{d} x \quad \xi \in \widehat{\mathbb{R}}^{2}
$$

whenever $f \in L^{1}\left(\mathbb{R}^{2}\right) \cap L^{2}\left(\mathbb{R}^{2}\right)$ and $x \cdot \xi$ is the Euclidean scalar product.

Define the unitary operator $J: L^{2}\left(\widehat{\mathbb{R}}^{2}\right) \rightarrow L^{2}\left(\widehat{\mathbb{R}}_{+} \times \mathcal{T}\right)$ by

$$
J \hat{f}(\omega, \theta)=\hat{f}(\sqrt{\omega} \cos \theta, \sqrt{\omega} \sin \theta) / \sqrt{2} \quad \hat{f} \in L^{2}\left(\widehat{\mathbb{R}}^{2}\right), \omega \in \widehat{\mathbb{R}}_{+}, \theta \in \mathcal{T} \text {. }
$$

The unitarily equivalent representation $(J \mathcal{F}) \pi(J \mathcal{F})^{-1}$ acting on $L^{2}\left(\widehat{\mathbb{R}}_{+} \times \mathcal{T}\right)$ reads

$$
(J \mathcal{F}) \pi(J \mathcal{F})^{-1}(b, a, \phi) \hat{f}(\omega, \theta)=a^{1 / 2} e^{-2 \pi i b \omega} \hat{f}(a \omega, \theta-\phi) \quad \omega \in \widehat{\mathbb{R}}_{+}, \theta \in \mathcal{T}
$$

for all $(b, a, \phi) \in G$ and $\hat{f} \in L^{2}\left(\widehat{\mathbb{R}}_{+} \times \mathcal{T}\right)$. The action on the radial variable can be described by the representation of $\mathbb{R} \rtimes \mathbb{R}_{+}$on $L^{2}\left(\widehat{\mathbb{R}}_{+}\right)$given by

$$
\widehat{W}^{+}(b, a) g(\omega)=a^{1 / 2} e^{-2 \pi i b \omega} g(a \omega) \quad \omega \in \widehat{\mathbb{R}}_{+},(b, a) \in \mathbb{R} \rtimes \mathbb{R}_{+}, g \in L^{2}\left(\widehat{\mathbb{R}}_{+}\right),
$$

which is nothing else than the one-dimensional wavelet representation in the positive frequency domain. The action on the angular variable is simply given by a rotation $\rho(\phi) z(\theta)=z(\theta-\phi)$ for $z \in L^{2}(\mathcal{T})$. Therefore, the action of $\pi$ on two-dimensional functions should be thought of as a classical one-dimensional wavelet representation on the radial component combined with rotations around the origin.

Consider now the Fourier series with respect to $\theta$ and define the unitary operator $S: L^{2}\left(\mathbb{R}^{2}\right) \rightarrow \bigoplus_{n \in \mathbb{Z}} L^{2}\left(\widehat{\mathbb{R}}_{+}\right)$by

$$
(S f)_{n}(\omega)=\int_{0}^{2 \pi}(J \mathcal{F} f)(\omega, \theta) e^{-i n \theta} \frac{\mathrm{d} \theta}{\sqrt{2 \pi}} \quad \omega \in \widehat{\mathbb{R}}_{+}, n \in \mathbb{Z}, f \in L^{2}\left(\mathbb{R}^{2}\right) .
$$

From now on, we shall consider the equivalent representation $\pi^{\prime}=S \pi S^{-1}$ of $G$ acting on $\bigoplus_{n \in \mathbb{Z}} L^{2}\left(\widehat{\mathbb{R}}_{+}\right)$. In view of (2.1) and (2.2), the action of $\pi^{\prime}$ is given by

$$
\left(\pi^{\prime}(b, a, \phi) \hat{f}\right)_{n}=e^{-i n \phi}\left(\widehat{W}^{+}(b, a) \hat{f}_{n}\right) \quad n \in \mathbb{Z}, \hat{f} \in \bigoplus_{n \in \mathbb{Z}} L^{2}\left(\widehat{\mathbb{R}}_{+}\right),(b, a, \phi) \in G .
$$

Denote the character $\phi \mapsto e^{-i n \phi}$ of $\mathcal{T}$ by $\rho_{n}$; the representation $\pi^{\prime}$ can be decomposed as

$$
\pi^{\prime}=\bigoplus_{n \in \mathbb{Z}} \rho_{n} \widehat{W}^{+}
$$

where each component $\rho_{n} \widehat{W}^{+}$acts irreducibly on $L^{2}\left(\widehat{\mathbb{R}}_{+}\right)$.

It was proven in [2, 19] that $\pi^{\prime}$, and therefore $\pi$, is reproducing, namely

$$
\|\hat{f}\|_{\bigoplus_{n} L^{2}\left(\widehat{\mathbb{R}}_{+}\right)}^{2}=\int_{G}\left|\left\langle\pi^{\prime}(b, a, \phi) \hat{\eta}, \hat{f}\right\rangle\right|^{2} \mathrm{~d} b \frac{\mathrm{d} a}{a^{2}} \frac{\mathrm{d} \phi}{2 \pi} \quad \hat{f} \in \bigoplus_{n \in \mathbb{Z}} L^{2}\left(\widehat{\mathbb{R}}_{+}\right)
$$


for some admissible vector $\widehat{\eta} \in \bigoplus_{n \in \mathbb{Z}} L^{2}\left(\widehat{\mathbb{R}}_{+}\right)$. A vector $\widehat{\eta}=\left(\widehat{\eta}_{n}\right)_{n} \in \bigoplus_{n \in \mathbb{Z}} L^{2}\left(\widehat{\mathbb{R}}_{+}\right)$ is admissible for $\pi^{\prime}$ if and only if

$$
\int_{0}^{+\infty}\left|\widehat{\eta}_{n}(\omega)\right|^{2} \frac{\mathrm{d} \omega}{\omega}=1 \quad n \in \mathbb{Z}
$$

namely, if and only if each component $\widehat{\eta}_{n}$ is a one-dimensional wavelet 20. A simple way to construct admissible vectors in $\bigoplus_{n \in \mathbb{Z}} L^{2}\left(\widehat{\mathbb{R}}_{+}\right)$satisfying (2.4) is to fix a one-dimensional wavelet $\widehat{\eta}_{0} \in L^{2}\left(\widehat{\mathbb{R}}_{+}\right)$satisfying (2.4) and then construct all the other components $\widehat{\eta}_{n}$ by dilating $\widehat{\eta}_{0}$. Since (2.4) is invariant under positive dilations, it is immediately satisfied for all $n$. More precisely, set for all $n \in \mathbb{Z}$

$$
\widehat{\eta}_{n}(\omega)=\widehat{\eta}_{0}\left(\alpha_{n}^{-1} \omega\right) \quad \omega \in \widehat{\mathbb{R}}_{+},
$$

for some weights $\alpha_{n}>0$ that satisfy $\alpha_{0}=1$ and $\sum_{n} \alpha_{n}<\infty$. This last condition ensures that the resulting $\widehat{\eta}$ has finite norm in $\bigoplus_{n \in \mathbb{Z}} L^{2}\left(\widehat{\mathbb{R}}_{+}\right)$, because

$$
\|\widehat{\eta}\|^{2}=\left\|\widehat{\eta}_{0}\right\|_{L^{2}\left(\widehat{\mathbb{R}}_{+}\right)}^{2} \sum_{n} \alpha_{n} .
$$

2.2. The discrete Schrödingerlets in 2D. We now show how to construct a Parseval frame of $\bigoplus_{n \in \mathbb{Z}} L^{2}\left(\widehat{\mathbb{R}}_{+}\right)$associated to $\pi^{\prime}$. Then, by means of the intertwining operator $S$, this frame can be transformed into a Parseval frame of $L^{2}\left(\mathbb{R}^{2}\right)$ associated to $\pi$. Constructing a Parseval frame corresponds to a discretization of (2.3) of the form

$$
\|\hat{f}\|_{\oplus_{n} L^{2}\left(\widehat{\mathbb{R}}_{+}\right)}^{2}=\sum_{i \in \mathbb{N}}\left|\left\langle\pi^{\prime}\left(x_{i}\right) \widehat{\eta}, \hat{f}\right\rangle\right|^{2} \quad \hat{f} \in \bigoplus_{n \in \mathbb{Z}} L^{2}\left(\widehat{\mathbb{R}}_{+}\right),
$$

for suitable choices of the admissible vector $\widehat{\eta}$ and of a sampling $\left\{x_{i}\right\}_{i \in \mathbb{N}}$ of the group $G$.

Our approach is based on the fact that $\pi^{\prime}$ is the direct sum of wavelet representations acting on 1D signals. Thus, it is instructive to look first at the well known one-dimensional case, namely at the representation $\widehat{W}^{+}$acting on $L^{2}\left(\widehat{\mathbb{R}}_{+}\right)$. Standard wavelet theory [18, Thm. 1.1, Chapter 7] gives that $\left\{\widehat{W}^{+}\left(2^{j} k, 2^{j}\right) \widehat{\eta}_{0}: k, j \in \mathbb{Z}\right\}$ is a Parseval frame for $L^{2}\left(\widehat{\mathbb{R}}_{+}\right)$, namely

$$
\|\hat{f}\|_{L^{2}\left(\widehat{\mathbb{R}}_{+}\right)}^{2}=\sum_{k, j \in \mathbb{Z}}\left|\left\langle\widehat{W}^{+}\left(2^{j} k, 2^{j}\right) \widehat{\eta}_{0}, \hat{f}\right\rangle\right|^{2} \quad \hat{f} \in L^{2}\left(\widehat{\mathbb{R}}_{+}\right),
$$

provided that the conditions

$$
\begin{aligned}
& \sum_{j \in \mathbb{Z}}\left|\widehat{\eta}_{0}\left(2^{j} \omega\right)\right|^{2}=1, \quad \text { for a.e. } \omega \in \widehat{\mathbb{R}}_{+}, \\
& \sum_{j \in \mathbb{N}} \widehat{\eta}_{0}\left(2^{j} \omega\right) \overline{\widehat{\eta}_{0}\left(2^{j}(\omega+2 \pi m)\right)}=0, \quad \text { for a.e. } \omega \in \widehat{\mathbb{R}}_{+}, m \in 2 \mathbb{Z}+1
\end{aligned}
$$

hold true. Note that in this case the sampling of the group $\mathbb{R} \rtimes \mathbb{R}_{+}$is the discrete set $\left\{\left(2^{j} k, 2^{j}\right): k, j \in \mathbb{Z}\right\}$.

We now generalize this construction to the Schrödingerlets. In view of the above sampling of the affine group, it is natural to consider the discretization of $G$ given by

$$
\left\{x_{k, j, l}=\left(2^{j} k, 2^{j}, 2 \pi l / L\right): k, j \in \mathbb{Z}, l=0, \ldots, L-1\right\},
$$


for some $L \in \mathbb{Z}_{+}$, where $\mathbb{Z}_{+}:=\{1,2, \ldots\}$ is the set of positive integers. Note that the angles $\phi_{l}=2 \pi l / L$ give a uniform sampling of $\mathcal{T}$ and form a finite cyclic subgroup of order $L$. Let us now discuss suitable assumptions on the admissible vector $\widehat{\eta}$ so that $\left\{\pi^{\prime}\left(x_{k, j, l}\right) \widehat{\eta}: k, j \in \mathbb{Z}, l=0, \ldots, L-1\right\}$ is a Parseval frame for $\bigoplus_{n \in \mathbb{Z}} L^{2}\left(\widehat{\mathbb{R}}_{+}\right)$.

We first observe that for every $n \in \mathbb{Z}$ it is necessary that each $\widehat{\eta}_{n} \in L^{2}\left(\widehat{\mathbb{R}}^{+}\right)$ give rise to a Parseval frame for the corresponding space $L^{2}\left(\widehat{\mathbb{R}}_{+}\right)$, i.e. that each $\widehat{\eta}_{n}$ satisfies (2.6) (suitably normalized):

$$
\begin{aligned}
& \sum_{j \in \mathbb{Z}}\left|\widehat{\eta}_{n}\left(2^{j} \omega\right)\right|^{2}=1 / L, \quad \text { a.e. } \omega \in \widehat{\mathbb{R}}_{+}, n \in \mathbb{Z}, \\
& \sum_{j \in \mathbb{N}} \widehat{\eta}_{n}\left(2^{j} \omega\right) \overline{\widehat{\eta}_{n}\left(2^{j}(\omega+2 \pi m)\right)}=0, \quad \text { a.e. } \omega \in \widehat{\mathbb{R}}_{+}, n \in \mathbb{Z}, m \in 2 \mathbb{Z}+1 .
\end{aligned}
$$

In the continuous setting, it is necessary and sufficient to assume that each $\widehat{\eta}_{n}$ is a one-dimensional wavelet, i.e. that (2.4) holds true for every $n$, in order to have the continuous reproducing formula (2.3). In the discrete case, however, assumptions (2.7) are not sufficient, and we assume the following conditions to hold true:

$$
\sum_{j \in \mathbb{Z}} \widehat{\eta}_{n}\left(2^{j} \omega\right) \overline{\widehat{\eta}_{n+k L}\left(2^{j} \omega\right)}=0, \quad \text { a.e. } \omega \in \widehat{\mathbb{R}}_{+}, n \in \mathbb{Z}, k \in \mathbb{Z}^{*},
$$

$$
\sum_{j \in \mathbb{N}} \widehat{\eta}_{n}\left(2^{j} \omega\right) \overline{\widehat{\eta}_{n+k L}\left(2^{j}(\omega+2 \pi m)\right)}=0 \text {, a.e. } \omega \in \widehat{\mathbb{R}}_{+}, n \in \mathbb{Z}, k \in \mathbb{Z}^{*}, m \in 2 \mathbb{Z}+1
$$

where $\mathbb{Z}^{*}=\mathbb{Z} \backslash\{0\}$. When $\eta_{n}$ is given by (2.5), the above expressions can be simplified into conditions involving $\widehat{\eta}_{0}$ and the weights $\alpha_{n}$.

These orthogonality relations do not contain all the cross terms between $\widehat{\eta}_{n}$ and $\widehat{\eta}_{m}$ for $n \neq m$, but only those corresponding to the cases when $m-n \in L \mathbb{Z}$. The reason for this simplification can be explained as follows. Two characters $\rho_{n}$ and $\rho_{m}$ restricted to the finite subgroup $\{2 \pi l / L: l=0, \ldots, L-1\}$ are equivalent if and only if $m-n \in L \mathbb{Z}$. As a consequence, all the cross terms corresponding to $m$ and $n$ for which $m-n \notin L \mathbb{Z}$ are zero by Schur orthogonality relations for finite groups.

The following theorem shows that the above conditions are sufficient in order to obtain a Parseval frame.

Theorem 2.1. Let $\widehat{\eta} \in \bigoplus_{n \in \mathbb{Z}} L^{2}\left(\widehat{\mathbb{R}}_{+}\right)$be such that (2.7) and (2.8) hold true, and take $L \in \mathbb{Z}_{+}$. Then $\left\{\pi^{\prime}\left(x_{k, j, l}\right) \widehat{\eta}: k, j \in \mathbb{Z}, l=0, \ldots, L-1\right\}$ is a Parseval frame for $\bigoplus_{n \in \mathbb{Z}} L^{2}\left(\widehat{\mathbb{R}}_{+}\right)$, namely

$$
\|\hat{f}\|_{\oplus_{n} L^{2}\left(\widehat{\mathbb{R}}_{+}\right)}^{2}=\sum_{k, j, l}\left|\left\langle\pi^{\prime}\left(x_{k, j, l}\right) \widehat{\eta}, \hat{f}\right\rangle\right|^{2} \quad \hat{f} \in \bigoplus_{n \in \mathbb{Z}} L^{2}\left(\widehat{\mathbb{R}}_{+}\right) .
$$

The proof is given in Section 3.4 as part of the proof of Theorem 3.4. Here we just exhibit functions $\widehat{\eta}$ satisfying the assumptions. Take $\widehat{\eta}_{0} \in L^{2}\left(\widehat{\mathbb{R}}_{+}\right)$such that (2.7a) is satisfied for $n=0$ and such that supp $\widehat{\eta}_{0} \subseteq[0,2 \pi]$. Moreover, choose weights $\alpha_{n} \in(0,1]$ such that $\alpha_{0}=1, \sum_{n} \alpha_{n}<\infty$ and

$$
\left|\operatorname{supp}\left(\widehat{\eta}_{0}\right) \cap \alpha_{n}^{-1} \alpha_{n+k L} \operatorname{supp}\left(\widehat{\eta}_{0}\right)\right|=0 \quad n \in \mathbb{Z}, k \in \mathbb{Z}^{*},
$$


where $|\cdot|$ denotes Lebesgue measure. It is easy to see that the admissible vector $\widehat{\eta} \in \bigoplus_{n \in \mathbb{Z}} L^{2}\left(\widehat{\mathbb{R}}_{+}\right)$defined by (2.5) satisfies (2.7) and (2.8). A simple choice valid for any $L$ is $\widehat{\eta}_{0}=L^{-1} \chi_{[1 / 2,1]}$ and

$$
\alpha_{n}= \begin{cases}2^{-2 n} & \text { if } n \geq 0 \\ 2^{2 n+1} & \text { if } n<0 .\end{cases}
$$

We now comment on the role of the number of rotations $L$. The conclusion of Theorem 2.1 still holds true when $L=1$, namely when no rotations are considered. However, the rotations do play a role in the choice of the admissible vector $\widehat{\eta}$. Indeed, condition (2.8), or (2.9) in the case when (2.5) holds true, becomes weaker as $L$ increases. More precisely, if $L_{2}$ is a multiple of $L_{1}$ and $\widehat{\eta}$ satisfies (2.8) with $L=L_{1}$, then the same equalities hold true with $L=L_{2}$. Note that this is equivalent to saying that the two corresponding discrete subgroups of $\mathcal{T}$ are one contained into the other.

Note that for $L=1$ a simple computation shows that $\|\widehat{\eta}\|=1$, hence the frame obtained in Theorem 2.1 is in fact an orthonormal basis of $\bigoplus_{n \in \mathbb{Z}} L^{2}\left(\widehat{\mathbb{R}}_{+}\right)$. Indeed, it is a standard general fact that a tight frame whose elements have norm (greater than or equal to) one is necessarily an orthonormal basis (see e.g. 18, Theorem 1.8, Ch. 7]).

\section{The $d$-DIMENSIONAL CASE}

3.1. The continuous setting. We define $G=\left(\mathbb{R} \rtimes \mathbb{R}_{+}\right) \times \mathrm{SO}(d)$ as the direct product of the identity component of the one-dimensional affine group and $\mathrm{SO}(d)$. Clearly, the set

$$
H=\left\{(0, a, R) \mid a \in \mathbb{R}_{+}, R \in \mathrm{SO}(d)\right\} \simeq \mathbb{R}_{+} \times \mathrm{SO}(d)
$$

is a closed unimodular subgroup of $G$ and its Haar measure is $\mathrm{d} h=a^{-1} \mathrm{~d} a \mathrm{~d} R$, and the set

$$
\{(b, 1, \mathrm{I}) \mid b \in \mathbb{R}\} \simeq \mathbb{R}
$$

is a normal abelian closed subgroup of $G$, whose Haar measure is the Lebesgue measure d $b$. Moreover, $G$ is the semi-direct product of $\mathbb{R}$ and $H$ with respect to the inner action of $H$ on $\mathbb{R}$ given by

$$
h[b]=a b \quad b \in \mathbb{R}, h=(a, R) \in H .
$$

We set

$$
\gamma(h)=\operatorname{det}(b \mapsto h[b])=a .
$$

The Schrödinger representation $\pi$ of $G$ acts on $L^{2}\left(\mathbb{R}^{d}\right)$ as

$$
\pi(b, a, R)=U(b) V(a, R) \quad(b, a, R) \in G .
$$

Here $V(a, R)$ is the unitary operator

$$
V(a, R) f(x)=a^{-\frac{d}{4}} f\left(a^{-\frac{1}{2}} R^{-1} x\right) \quad f \in L^{2}\left(\mathbb{R}^{d}\right), x \in \mathbb{R}^{d},
$$

and $b \mapsto U(b)$ is the one-parameter group of unitary operators on $L^{2}\left(\mathbb{R}^{d}\right)$ associated with the Laplacian by the spectral calculus, namely

$$
U(b)=e^{i \frac{b}{2 \pi} \Delta} .
$$

Thus

$$
\mathcal{F} U(b) \mathcal{F}^{-1} \hat{f}(\xi)=e^{-2 \pi i b \xi \cdot \xi} \hat{f}(\xi) \quad \xi \in \widehat{\mathbb{R}}^{d} .
$$


Setting $\widehat{\pi}=\mathcal{F} \pi \mathcal{F}^{-1}$ we get

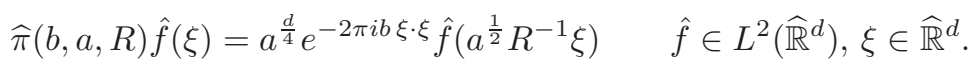

We now prove that $\pi$ is a reproducing representation.

Proposition 3.1. The Schrödinger representation $\pi$ of $G$ is a reproducing representation.

Proof. It is enough to prove the result for $\widehat{\pi}$, which belongs to the family of representations introduced in [3], regarding $G$ as semi-direct product of $\mathbb{R}$ and $H$. Indeed, $H$ acts on the dual group $\widehat{\mathbb{R}}$ of $\mathbb{R}$ by the contra-gradient action

$$
{ }^{t} h[\omega]=a^{-1} \omega \quad \omega \in \widehat{\mathbb{R}}, h=(a, R) \in H .
$$

The group $H$ acts on $\mathbb{R}^{d}$ as well as on the dual space $\widehat{\mathbb{R}}^{d}$ by means of

$$
\begin{aligned}
h . x & =a^{\frac{1}{2}} R x \\
{ }^{t} h . \xi & =a^{-\frac{1}{2}} R \xi
\end{aligned} \quad x \in \mathbb{R}^{d}, \xi \in \widehat{\mathbb{R}}^{d}, h=(a, R) \in H .
$$

We set

$$
\beta(h)=\operatorname{det}\left(\xi \mapsto{ }^{t} h \cdot \xi\right)=a^{-\frac{d}{2}} .
$$

The map

$$
\Phi: \widehat{\mathbb{R}}^{d} \longrightarrow \widehat{\mathbb{R}}, \quad \Phi(\xi)=\xi \cdot \xi
$$

is easily seen to satisfy the following properties:

i) $\Phi$ is a smooth map whose gradient is $\nabla \Phi(\xi)=2 \xi$;

ii) the set of critical points of $\Phi$ reduces to the origin, which is a Lebesgue negligible set, and $\Phi\left(\widehat{\mathbb{R}}^{d} \backslash\{0\}\right)=\widehat{\mathbb{R}}_{+}$;

iii) $\Phi\left({ }^{t} h . \xi\right)={ }^{t} h[\Phi(\xi)]$ for all $\xi \in \widehat{\mathbb{R}}^{d}$ and $h \in H$;

iv) the action of $H$ on $\widehat{\mathbb{R}}_{+}$is transitive, the stability subgroup at $1 \in \mathbb{R}_{+}$is the compact group $\mathrm{SO}(d)$, and $q:(0,+\infty) \rightarrow H, q(\omega)=\omega^{-1}$, is a smooth section, namely

$$
{ }^{t} q(\omega)[1]=\omega \quad \omega \in \mathbb{R}_{+} ;
$$

v) $\Phi^{-1}(1)=\mathrm{S}^{d-1}$, where $\mathrm{S}^{d-1}$ is the unit sphere of $\widehat{\mathbb{R}}^{d}$ endowed with the Riemannian measure $\mathrm{d} s$.

From (3.2d) it is clear that

$$
\widehat{\pi}(b, h) \hat{f}(\xi)=\beta(h)^{-\frac{1}{2}} e^{-2 \pi i b \Phi(\xi)} \hat{f}\left({ }^{t} h^{-1} \cdot \xi\right),
$$

where $\xi \in \widehat{\mathbb{R}}^{d}, \hat{f} \in L^{2}\left(\widehat{\mathbb{R}}^{d}\right)$ and $(b, R) \in \mathbb{R} \rtimes\left(\mathbb{R}_{+} \times \mathrm{SO}(d)\right)$, which shows that $\widehat{\pi}$ is the mock-metaplectic representation associated with the map $\Phi$ (see [3]). Theorem 9 of [3] then implies that $\widehat{\pi}$ is a reproducing representation.

It is worth noting that the above proof works also in the case when $G$ is simply given by $\mathbb{R} \rtimes \mathbb{R}_{+}$, namely in absence of rotations. Indeed, the only difference is in the stability subgroup at 1 , which would be trivial in this case. Therefore, the corresponding representation is reproducing also without considering rotations.

We now study the admissible vectors of $\pi$. First, we need to recall some elementary facts.

Let $\rho$ be the regular representation of $\mathrm{SO}(d)$ acting on $L^{2}\left(\mathrm{~S}^{d-1}\right)$, namely

$$
\rho(R) \varphi(s)=\varphi\left(R^{-1} s\right) \quad s \in \mathrm{S}^{d-1}, \varphi \in L^{2}\left(\mathrm{~S}^{d-1}\right), R \in \mathrm{SO}(d) .
$$


There holds that

$$
L^{2}\left(\mathrm{~S}^{d-1}\right)=\bigoplus_{i \in \mathbb{N}} \mathcal{H}_{i}
$$

where each $\mathcal{H}_{i}$ is the space of spherical harmonics, namely the complex polynomials in $d$ variables, homogeneous of degree $i$ and harmonic. Here each polynomial is regarded as a function on $\mathrm{S}^{d-1}$, so that $\mathcal{H}_{i}$ can be identified as a subspace of $L^{2}\left(\mathrm{~S}^{d-1}\right)$. For an account of the role of spherical harmonics in the representation theory of the orthogonal groups see [21]. Let $d_{i}:=\operatorname{dim} \mathcal{H}_{i}$. It is known that

$$
d_{0}=1, \quad d_{1}=d, \quad d_{i}=\left(\begin{array}{c}
d+i-1 \\
d-1
\end{array}\right)-\left(\begin{array}{c}
d+i-3 \\
d-1
\end{array}\right), i \geq 2 .
$$

Moreover,

$$
\rho=\bigoplus_{i \in \mathbb{N}} \rho_{i},
$$

where $\rho_{i}$ is the restriction of $\rho$ to $\mathcal{H}_{i}$. We denote by $P_{i}$ the projection from $L^{2}\left(\mathrm{~S}^{d-1}\right)$ onto $\mathcal{H}_{i}$.

If $d>2$, each representation $\rho_{i}$ is irreducible, and two representations $\rho_{i}$ and $\rho_{j}$ are inequivalent whenever $i \neq j$ (the multiplicity of each $\rho_{i}$ is one). For $d=2$, every $\mathcal{H}_{i}$ with $i \geq 1$ has dimension 2 and each $\rho_{i}$ is the sum of two inequivalent irreducible one-dimensional representations, namely $\rho_{i}^{+}(\theta)=e^{i n \theta}, \rho_{i}^{-}(\theta)=e^{-i n \theta}$, $\theta \in \mathrm{SO}(2) \simeq \mathcal{T}$. Hence, we still obtain a decomposition into inequivalent irreducible representations if we just replace the index set $\mathbb{N}$ with $\mathbb{Z}$. For simplicity, we shall proceed using the notation of the case $d \geq 3$. Except for this minor notational difference, the two-dimensional case described in Section 2 is completely covered by the argument given below.

Recall that the group $\mathbb{R} \rtimes \mathbb{R}_{+}$has only two inequivalent infinite dimensional irreducible representations up to unitary equivalence, which we denote by $\widehat{W}^{+}$and $\widehat{W}^{-}$(see e.g. [22]). Each of them acts on $L^{2}\left(\widehat{\mathbb{R}}_{+}\right)$and $L^{2}\left(\widehat{\mathbb{R}}_{-}\right)$, respectively, as

$$
\widehat{W}^{ \pm}(b, a) \varphi(\omega)=a^{\frac{1}{2}} \varphi(a \omega) e^{-2 \pi i b \omega} \quad \omega \in \widehat{\mathbb{R}}_{ \pm},(b, a) \in \mathbb{R} \rtimes \mathbb{R}_{+},
$$

where $\varphi \in L^{2}\left(\widehat{\mathbb{R}}_{ \pm}\right)$.

Now, let $J: L^{2}\left(\widehat{\mathbb{R}}^{d}\right) \rightarrow L^{2}\left(\widehat{\mathbb{R}}_{+} \times \mathrm{S}^{d-1}\right)$ be the operator defined by

$$
J \hat{f}(\omega, s)=\frac{\omega^{\frac{d-2}{4}}}{\sqrt{2}} \hat{f}(\sqrt{\omega} s) \quad \omega \in \widehat{\mathbb{R}}_{+}, s \in \mathrm{S}^{d-1}, \hat{f} \in L^{2}\left(\widehat{\mathbb{R}}^{d}\right) .
$$

We have the following simple lemma.

Lemma 3.2. The operator $J$ is unitary.

Proof. If $\hat{f} \in L^{2}\left(\widehat{\mathbb{R}}^{d}\right)$, then the changes of variable $\omega=r^{2}$ and $\xi=r s$ yield

$$
\int_{\widehat{\mathbb{R}}_{+} \times \mathrm{S}^{d-1}} \omega^{\frac{d-2}{2}}|\hat{f}(\sqrt{\omega} s)|^{2} \frac{\mathrm{d} \omega \mathrm{d} s}{2}=\int_{\widehat{\mathbb{R}}_{+} \times S^{d-1}} r^{d-1}|\hat{f}(r s)|^{2} \mathrm{~d} r \mathrm{~d} s=\int_{\widehat{\mathbb{R}}^{d}}|\hat{f}(\xi)|^{2} \mathrm{~d} \xi .
$$

The inverse of $J$ is given by

$$
\left(J^{-1} g\right)(\xi)=\frac{\sqrt{2}}{(\xi \cdot \xi)^{d-2}} g\left(\xi \cdot \xi, \frac{\xi}{\sqrt{\xi \cdot \xi}}\right) \quad \xi \in \widehat{\mathbb{R}}^{d}, \xi \neq 0, g \in L^{2}\left(\widehat{\mathbb{R}}_{+} \times \mathrm{S}^{d-1}\right),
$$

which proves that $J$ is unitary. 
In what follows, we will freely identify

$$
\begin{aligned}
L^{2}\left(\widehat{\mathbb{R}}^{d}\right) & \simeq L^{2}\left(\widehat{\mathbb{R}}_{+} \times \mathrm{S}^{d-1}\right) \\
& \simeq L^{2}\left(\widehat{\mathbb{R}}_{+}\right) \otimes L^{2}\left(\mathrm{~S}^{d-1}\right) \\
& \simeq \bigoplus_{i \in \mathbb{N}} L^{2}\left(\widehat{\mathbb{R}}_{+}\right) \otimes \mathcal{H}_{i} \\
& \simeq \bigoplus_{i \in \mathbb{N}} L^{2}\left(\widehat{\mathbb{R}}_{+}, \mathcal{H}_{i}\right) .
\end{aligned}
$$

We define the unitary operator $S: L^{2}\left(\mathbb{R}^{d}\right) \rightarrow \bigoplus_{i \in \mathbb{N}} L^{2}\left(\widehat{\mathbb{R}}_{+}, \mathcal{H}_{i}\right)$ by

$$
(S f)_{i}=\left(\operatorname{Id} \otimes P_{i}\right)(J \mathcal{F} f) \quad f \in L^{2}\left(\mathbb{R}^{d}\right) .
$$

Proposition 3.3. With the above notation,

$$
S \pi S^{-1}=\bigoplus_{i \in \mathbb{N}} \widehat{W}^{+} \otimes \rho_{i}
$$

where each component $\widehat{W}^{+} \otimes \rho_{i}$ is irreducible and inequivalent to the others. A vector $\eta \in L^{2}\left(\mathbb{R}^{d}\right)$ is admissible for $\pi$ if and only if

$$
\int_{0}^{+\infty}\left\|(S \eta)_{i}(\omega)\right\|_{\mathcal{H}_{i}}^{2} \frac{\mathrm{d} \omega}{\omega}=d_{i} \quad i \in \mathbb{N} .
$$

Proof. This proon is based on the general theory developed in 3 . We sketch the main steps. For any $\omega \in \widehat{\mathbb{R}}_{+}$we denote by $\nu_{\omega}$ the measure on $\widehat{\mathbb{R}}^{d}$ which is the image measure of $\omega^{\frac{d-2}{2}} \mathrm{~d} s / 2$ under the map

$$
\mathrm{S}^{d-1} \ni s \mapsto \sqrt{\omega} s \in \widehat{\mathbb{R}}^{d},
$$

so that, for all compactly supported continuous functions $\varphi$, we have

$$
\int_{\widehat{\mathbb{R}}^{d}} \varphi(\xi) \mathrm{d} \nu_{\omega}(\xi)=\int_{\mathrm{S}^{d-1}} \varphi(\sqrt{\omega} s) \frac{\omega^{\frac{d-2}{2}}}{2} \mathrm{~d} s .
$$

The change of variable in spherical coordinates (as in (3.10) ) gives

$$
\begin{aligned}
\int_{\widehat{\mathbb{R}}^{d}} \varphi(\xi) \mathrm{d} \xi & =\int_{0}^{+\infty}\left(\int_{\mathrm{S}^{d-1}} \varphi(r s) r^{d-1} \mathrm{~d} s\right) \mathrm{d} r \\
& =\int_{0}^{+\infty}\left(\int_{\mathrm{S}^{d-1}} \varphi(\sqrt{\omega} s) \frac{\omega^{\frac{d-2}{2}}}{2} \mathrm{~d} s\right) \mathrm{d} \omega \\
& =\int_{0}^{+\infty}\left(\int_{\widehat{\mathbb{R}}^{d}} \varphi(\xi) \mathrm{d} \nu_{\omega}(\xi)\right) \mathrm{d} \omega,
\end{aligned}
$$

where $r^{2}=\omega$, so that the disintegration formula

$$
\mathrm{d} \xi=\int_{0}^{+\infty} \nu_{\omega} \mathrm{d} \omega
$$

holds true. Finally, Weil's formula for quasi-invariant measure on quotient spaces [5] reads

$$
\int_{H} \varphi(a, R) \gamma(a)^{-1} \frac{\mathrm{d} a}{a} \mathrm{~d} R=C \int_{0}^{+\infty}\left(\int_{\mathrm{SO}(d)} \varphi(q(\omega) R) \mathrm{d} R\right) \mathrm{d} \omega
$$

\footnotetext{
${ }^{1}$ An alternative proof can be derived using Proposition 2.23 of [5].
} 
for some constant $C$, to be computed. Recalling (3.1) and $q(\omega)=\omega^{-1}$, we obtain $C=1$ since

$$
\int_{0}^{+\infty}\left(\int_{\mathrm{SO}(d)} \varphi\left(\omega^{-1} R\right) \mathrm{d} R\right) \mathrm{d} \omega=\int_{0}^{+\infty}\left(\int_{\mathrm{SO}(d)} \varphi(\omega, R) \mathrm{d} R\right) \frac{\mathrm{d} \omega}{\omega^{2}} .
$$

Observe that

i) $L^{2}\left(\widehat{R}^{d}, 2 \nu_{1}\right) \simeq L^{2}\left(\mathrm{~S}^{d-1}\right)$;

ii) the "restriction" of the mock-metaplectic representation $\widehat{\pi}$ to the fiber $\Phi^{-1}(1)$ and to the stability subgroup $\mathrm{SO}(d)$ is precisely $\rho$. Hence, (3.7) provides the decomposition of $\rho$ into its irreducibles, all of them with multiplicity 1 ;

iii) up to the normalization factor $1 / \sqrt{2}$, the operator $S \mathcal{F}^{-1}$ coincides with the operator introduced in 3 , whose main feature is that it decomposes $\widehat{\pi}$ into its irreducibles, each of which is the canonical representation obtained by inducing the irreducible representation of $\mathbb{R} \times \mathrm{SO}(d)$ acting on $\mathcal{H}_{i}$ as

$$
(b, R) \mapsto e^{-2 \pi i b} \rho_{i}(R)
$$

from $\mathbb{R} \times \mathrm{SO}(d)$ to $G$.

Theorem 9 of $\left[3\right.$ shows that $\eta \in L^{2}\left(\mathbb{R}^{d}\right)$ is admissible if and only if, for all $i \in \mathbb{N}$,

$$
\int_{0}^{+\infty}\left\|(S \eta)_{i}(\omega)\right\|_{\mathcal{H}_{i}}^{2} \gamma(q(\omega)) \mathrm{d} \omega=\frac{\operatorname{dim} \mathcal{H}_{i}}{C}=d_{i} .
$$

Explicitly, this amounts to

$$
\int_{0}^{+\infty}\left\|(S \eta)_{i}(\omega)\right\|_{\mathcal{H}_{i}}^{2} \frac{\mathrm{d} \omega}{\omega}=d_{i}
$$

We mentioned above after Proposition 3.1 that the reproducing property holds true also in absence of rotations. The same is true for the construction of the admissible vectors given in Proposition 3.3 Indeed, it is enough to let $\rho$ be the trivial representation of the identity group and to substitute identity (3.5) with

$$
L^{2}\left(\mathrm{~S}^{d-1}\right)=\bigoplus_{i \in \mathbb{N}} \mathcal{H}_{i}, \quad \mathcal{H}_{i}=\operatorname{span}\left\{h_{i}\right\}
$$

where $\left\{h_{i}\right\}_{i}$ is an orthonormal basis of $L^{2}\left(\mathrm{~S}^{d-1}\right)$, so that expression (3.7) still provides the decomposition of $\rho$ into its irreducibles. The rest of the derivation is identical as above. As it is natural, the corresponding conditions (3.14) become stronger in this case, which is the price to pay for the removal of the rotations. This is the continuous counterpart of what observed on the role of rotations for the construction of a Parseval frame (see the comments after Theorem 2.1).

3.2. A family of admissible vectors. We now show a procedure to construct all the admissible vectors $\eta \in L^{2}\left(\mathbb{R}^{d}\right)$ based on the identification provided by (3.11). Let $\eta \in L^{2}\left(\mathbb{R}^{d}\right)$ satisfy (3.14).

For any fixed $i \in \mathbb{N}$, we choose an orthonormal basis $\left\{e_{i, k}\right\}_{k=1}^{d_{i}}$ of $\mathcal{H}_{i}$, and define $\varphi_{i, k}: \widehat{\mathbb{R}}_{+} \rightarrow \mathbb{C}$ by

$$
\varphi_{i, k}(\omega)=\left\langle(S \eta)_{i}(\omega), e_{i, k}\right\rangle_{\mathcal{H}_{i}}
$$


so that

$$
(S \eta)_{i}=\sum_{k=1}^{d_{i}} \varphi_{i, k} \otimes e_{i, k}
$$

By construction, $\varphi_{i, k} \in L^{2}\left(\widehat{\mathbb{R}}_{+}\right)$. Furthermore, condition 3.14 implies

$$
c_{i, k}^{2}:=\int_{0}^{+\infty} \frac{\left|\varphi_{i, k}(\omega)\right|^{2}}{\omega} \mathrm{d} \omega<+\infty .
$$

If $\varphi_{i, k} \neq 0$, replacing $e_{i, k}$ with $v_{i, k}=c_{i, k} e_{i, k}$ in (3.17) we can always assume that

$$
\int_{0}^{+\infty} \frac{\left|\varphi_{i, k}(\omega)\right|^{2}}{\omega} \mathrm{d} \omega=1,
$$

i.e. $\varphi_{i, k}$ is a $1 D$-wavelet for $\widehat{W}^{+}$. Hence

$$
(S \eta)_{i}=\sum_{k=1}^{d_{i}} \varphi_{i, k} \otimes v_{i, k},
$$

where $\left\{v_{i, k}\right\}_{k=1}^{d_{i}}$ is an orthogonal family in $\mathcal{H}_{i}$ such that

$$
\sum_{k=1}^{d_{i}}\left\|v_{i, k}\right\|_{\mathcal{H}_{i}}^{2}=d_{i}
$$

and all $\varphi_{i, k}$ satisfy (3.18a). If for some $k$ the function $\left\langle(S \eta)_{i}(\cdot), e_{i, k}\right\rangle_{\mathcal{H}_{i}}$ is zero, we set $v_{i, k}=0$ and choose an arbitary $\varphi_{i, k}$ satisfying (3.18a).

The fact that $\eta \in L^{2}\left(\mathbb{R}^{d}\right)$ implies

$$
\sum_{i=1}^{+\infty} \sum_{k=1}^{d_{i}}\left\|\varphi_{i, k}\right\|_{2}^{2}\left\|v_{i, k}\right\|_{\mathcal{H}_{i}}^{2}<+\infty .
$$

Conversely, given a family $\left(\varphi_{i, k}, v_{i, k}\right)_{i \in \mathbb{N}, k=1, \ldots, d_{i}}$ such that

a) each $\varphi_{i, k}$ is in $L^{2}\left(\widehat{\mathbb{R}}_{+}\right)$and satisfies (3.18a $)$,

b) each family $\left\{v_{i, k}\right\}_{k=1}^{d_{i}}$ is orthogonal in $\mathcal{H}_{i}$ and satisfies (3.18c) and (3.18d),

then $\left(\varphi_{i, k}, v_{i, k}\right)_{i \in \mathbb{N}, k=1, \ldots, d_{i}}$ defines an admissible vector via (3.18b). A simple solution is given as follows. Choose a $1 \mathrm{D}$ wavelet $\varphi \in L^{2}\left(\widehat{\mathbb{R}}_{+}\right)$. For all $i \in \mathbb{N}$, fix $\alpha_{i}>0$ and $v_{i} \in \mathcal{H}_{i}$ with

$$
\left\|v_{i}\right\|_{\mathcal{H}_{i}}^{2}=d_{i}
$$

and

$$
\sum_{i \in \mathbb{N}} \alpha_{i} d_{i}<+\infty
$$

Define

$$
\varphi_{i}(\omega)=\varphi\left(\alpha_{i}^{-1} \omega\right) .
$$

Then, the vector $\eta \in L^{2}\left(\mathbb{R}^{d}\right)$ such that

$$
(S \eta)_{i}=\varphi_{i} \otimes v_{i}
$$

is admissible. 
3.3. Discretization. The aim of this section is to construct a Parseval frame for $L^{2}\left(\mathbb{R}^{d}\right)$ based on a discretization of the reproducing representation $\pi$.

We fix a finite subgroup of $\mathrm{SO}(d)$ of cardinality $L$

$$
F=\left\{R_{1}, \ldots, R_{L}\right\},
$$

and we choose as grid points those in the family

$$
x_{j, k, \ell}=\left(2^{j} k, 2^{j}, R_{\ell}\right) \quad j, k \in \mathbb{Z}, \ell=1, \ldots, L .
$$

We denote by $\widehat{F}$ the set of equivalence classes of irreducible (unitary) representations of $F$, and for each equivalence class in $\widehat{F}$ we fix a representative $\chi: F \rightarrow \mathcal{U}\left(\mathcal{H}_{\chi}\right)$, where $\mathcal{H}_{\chi}$ is the Hilbert space on which $\chi$ acts and $\mathcal{U}\left(\mathcal{H}_{\chi}\right)$ is the corresponding set of unitary operators. The dimension of $\mathcal{H}_{\chi}$, which is always finite, is denoted by $d_{\chi}$.

For each $i \in \mathbb{N}$, the representation $\rho_{i}$ restricted to $F$ decomposes into its irreducibles

$$
\mathcal{H}_{i}=\bigoplus_{\chi \in \widehat{F}} \mathcal{H}_{\chi} \otimes \mathbb{C}^{m_{i, \chi}} \quad \rho_{i \mid F}=\bigoplus_{\chi \in \widehat{F}} \chi \otimes \mathrm{I}_{m_{i, \chi}}
$$

where $m_{i, \chi} \in \mathbb{N}$ is the multiplicity of $\chi$ into $\rho_{i \mid F}$ (with the convention that $\mathbb{C}^{0}=\{0\}$ if $m_{i, \chi}=0$, namely when the representation $\chi$ does not enter into the decomposition).

We remark that in the two-dimensional case the picture is clearer (see Section 2 and the remarks that follow (3.7)). Taking $F=\{2 \pi l / L: l=0, \ldots, L-1\}$, the set $\widehat{F}$ is given by $L$ one-dimensional representations corresponding to the $L$-roots of unity, namely $\widehat{F}=\left\{\chi_{l}(\cdot)=e^{2 \pi i l \cdot / L}: l=0, \ldots, L-1\right\}$. Writing $\mathcal{H}_{k}=\operatorname{span}\left\{e^{i k \cdot}\right\}$ for $k \in \mathbb{Z}$ (as already observed, the natural index set in $2 \mathrm{D}$ is $\mathbb{Z}$ ), a simple calculation shows that $\rho_{k}$ corresponds to $\chi_{\bar{k}}$, where $\bar{k}=k \bmod L$. Therefore, in the above decomposition one has

$$
m_{k, \chi_{l}}= \begin{cases}1 & \text { if } k-l \in L \mathbb{Z} \\ 0 & \text { otherwise }\end{cases}
$$

or, equivalently, $\mathcal{H}_{k}=\mathcal{H}_{\chi_{\bar{k}}}$.

Back to the case $d \geq 3$, from (3.5) and (3.19) we finally obtain the decomposition of $\rho_{\mid F}$ into its irreducibles

$$
L^{2}\left(\mathrm{~S}^{d-1}\right)=\bigoplus_{\chi \in \widehat{F}} \mathcal{H}_{\chi} \otimes \mathbb{C}^{m_{\chi}} \quad \rho_{\mid F}=\bigoplus_{\chi \in \widehat{F}} \chi \otimes \mathrm{I}_{m_{\chi}}
$$

where $m_{\chi}=\sum_{i \in \mathbb{N}} m_{i, \chi}$, the operator $\mathrm{I}_{m_{\chi}}$ is the identity on $\mathbb{C}^{m_{\chi}}$ and $\mathbb{C}^{\infty}=\ell_{2}(\mathbb{N})$ if $\sum_{i \in \mathbb{N}} m_{i, \chi}=\infty$. By (3.11) and (3.20), the following identifications hold true:

$$
L^{2}\left(\widehat{\mathbb{R}}^{d}\right)=\bigoplus_{i \in \mathbb{N}, \chi \in \widehat{F}} L^{2}\left(\widehat{\mathbb{R}}_{+}, \mathcal{H}_{\chi}\right) \otimes \mathbb{C}^{m_{i, \chi}}=\bigoplus_{i \in \mathbb{N}, \chi \in \widehat{F}} \bigoplus_{\mu=1}^{m_{i, \chi}} L^{2}\left(\widehat{\mathbb{R}}_{+}, \mathcal{H}_{\chi} \otimes \mathbb{C}\left\{\epsilon_{\mu}\right\}\right)
$$

where $\left(\epsilon_{\mu}\right)_{\mu \in \mathbb{N}}$ is the canonical basis of $\ell^{2}(\mathbb{N})$ and each $\mathbb{C}^{m_{i, \chi}}$ is regarded as a closed subspace of $\ell^{2}(\mathbb{N})$. According to this decomposition, we denote by $P_{i, \chi, \mu}$ the orthogonal projection from $L^{2}\left(\widehat{\mathbb{R}}^{d}\right)$ onto the closed subspace $L^{2}\left(\widehat{\mathbb{R}}_{+}, \mathcal{H}_{\chi} \otimes \mathbb{C}\left\{\epsilon_{\mu}\right\}\right)$ of $L^{2}\left(\widehat{\mathbb{R}}_{+}, \mathcal{H}_{i}\right)$. 
Next, for each $\chi$, we select an orthogonal basis $w_{1}^{\chi}, \ldots, w_{d_{\chi}}^{\chi}$ in $\mathcal{H}_{\chi}$ such that

$$
\left\|w_{\delta}^{\chi}\right\|^{2}=d_{\chi} \quad \delta=1, \ldots, d_{\chi} .
$$

For each $i \in \mathbb{N}$, we choose $m_{i, \chi}$ vectors in this family and denote by $\Delta_{i, \chi}=$ $\left(\delta_{1}, \ldots, \delta_{m_{i, \chi}}\right)$ the corresponding family of indices (since any element $w_{\delta}^{\chi}$ can chosen many times, it can happen that $\delta_{\mu}=\delta_{\mu^{\prime}}$ for some pair of indices). We set

$$
v_{i, \chi, \mu}=w_{\delta_{\mu}}^{\chi} \otimes \epsilon_{\mu} \quad \mu=1, \ldots, m_{i, \chi},
$$

where each $v_{i, \chi, \mu}$ is a vector in $\mathcal{H}_{i}$ by means of (3.19).

Finally, we select $m_{i, \chi}$ functions $\varphi_{i, \chi, 1}, \ldots, \varphi_{i, \chi, m_{i, \chi}} \in L^{2}\left(\widehat{\mathbb{R}}_{+}\right)$such that the following conditions hold true:

a) the series

$$
\sum_{i \in \mathbb{N}} \sum_{\chi \in \hat{F}} d_{\chi}\left(\sum_{\mu=1}^{m_{i, \chi}}\left\|\varphi_{i, \chi, \mu}\right\|_{2}^{2}\right)<+\infty ;
$$

b) for each $i \in \mathbb{N}, \chi \in \widehat{F}$ and $\mu=1, \ldots, m_{i, \chi}$

$$
\sum_{j \in \mathbb{Z}}\left|\varphi_{i, \chi, \mu}\left(2^{j} \omega\right)\right|^{2}=\frac{1}{L} \quad \text { a.e. } \omega \in \widehat{\mathbb{R}}_{+},
$$

and for all odd integers $m$

$$
\sum_{j=0}^{+\infty} \varphi_{i, \chi, \mu}\left(2^{j} \omega\right) \overline{\varphi_{i, \chi, \mu}\left(2^{j}(\omega+2 \pi m)\right)}=0 \quad \text { a.e. } \omega \in \widehat{\mathbb{R}}_{+} ;
$$

c) for all $\chi \in \widehat{F}$, if there exists $i, i^{\prime} \in \mathbb{N}$ and $\mu=1, \ldots, m_{i, \chi}, \mu^{\prime}=1, \ldots, m_{i^{\prime}, \chi}$ such that $(i, \mu) \neq\left(i^{\prime}, \mu^{\prime}\right)$, but $w_{\delta_{\mu}}^{\chi}=w_{\delta_{\mu^{\prime}}}^{\chi}\left(\right.$ where $\delta_{\mu} \in \Delta_{i, \chi}$ and $\left.\delta_{\mu^{\prime}} \in \Delta_{i^{\prime}, \chi}\right)$, then

$$
\sum_{j \in \mathbb{Z}} \varphi_{i, \chi, \mu}\left(2^{j} \omega\right) \overline{\varphi_{i^{\prime}, \chi, \mu^{\prime}}\left(2^{j} \omega\right)}=0 \quad \text { a.e. } \omega \in \widehat{\mathbb{R}}_{+},
$$

and for all odd integers $m$

$$
\sum_{j=0}^{+\infty} \varphi_{i, \chi, \mu}\left(2^{j} \omega\right) \overline{\varphi_{i^{\prime}, \chi, \mu^{\prime}}\left(2^{j}(\omega+2 \pi m)\right)}=0 \quad \text { a.e. } \omega \in \widehat{\mathbb{R}}_{+} .
$$

Let us comment on the relation between these assumptions and the corresponding ones given in the two-dimensional case. Assumption (3.24) is simply a restatement of the fact that $\widehat{\eta}$ should have finite norm. Assumptions (3.25) and (3.26) correspond to assumptions (2.7) and (2.8), respectively. As we have already anticipated when discussing the $2 \mathrm{D}$ case, the condition $(i, \mu) \neq\left(i^{\prime}, \mu^{\prime}\right)$ corresponds to $m \neq n$ and $w_{\delta_{\mu}}^{\chi}=w_{\delta_{\mu^{\prime}}}^{\chi}$ corresponds to $m-n \in L \mathbb{Z}$.

We are now ready to state the main result of this paper.

Theorem 3.4. Let $\eta \in L^{2}\left(\mathbb{R}^{d}\right)$ be defined by

$$
(S \eta)_{i}=\sum_{\chi \in \widehat{F}} \sum_{\mu=1}^{m_{i, \chi}} \varphi_{i, \chi, \mu} \otimes v_{i, \chi, \mu} .
$$

Then the family $\left.\left\{\pi\left(2^{j} k, 2^{j}, R_{\ell}\right) \eta\right)\right\}_{j, k \in \mathbb{Z}, l=1, \ldots, L}$ is a Parseval frame for $L^{2}\left(\mathbb{R}^{d}\right)$. 
The proof is in Section 3.4. We add a few comments. Since $\sum_{\chi \in \widehat{F}} m_{i, \chi} d_{\chi}=d_{i}$, we have

$$
\sum_{\chi \in \widehat{F}} \sum_{\mu=1}^{m_{i, \chi}}\left\|v_{i, \chi, \mu}\right\|_{\mathcal{H}_{i}}^{2}=d_{i},
$$

hence (3.24) ensures that (3.27) is well defined (compare with (3.18b)).

An important result in wavelet theory [18, Theorem 1.6, Chapter 7] shows that (3.25a and 3.25b are equivalent to the fact that for each $i \in \mathbb{N}, \chi \in \widehat{F}$ and $\mu=1, \ldots, m_{i, \chi}$ the family $\left\{\widehat{W}^{+}\left(2^{j} k, 2^{j}\right) \sqrt{L} \varphi_{i, \chi, \mu}\right\}_{j, k \in \mathbb{Z}}$ is a Parseval frame for $L^{2}\left(\widehat{\mathbb{R}}_{+}\right)$. Furthermore, (3.25a) implies that

$$
\int_{\widehat{\mathbb{R}}_{+}} \frac{\left|\varphi_{i, \chi, \mu}(\omega)\right|^{2}}{\omega} \mathrm{d} \omega=\frac{\ln 2}{L},
$$

so that $\sqrt{L / \ln 2} \eta$ is an admissible vector for $\pi$ by Proposition 3.3 .

We now show that there exist families of $\left\{\varphi_{i, \chi, \mu}\right\}$, satisfying the above conditions. To this end, fix a function $\varphi \in L^{2}\left(\widehat{\mathbb{R}}_{+}\right)$supported in $[0,1]$ and such that

$$
\sum_{j \in \mathbb{Z}}\left|\varphi\left(2^{j} \omega\right)\right|^{2}=\frac{1}{L} \quad \text { a.e. } \omega \in \widehat{\mathbb{R}}_{+} .
$$

Choose a sequence $\left\{\alpha_{i, \chi, \mu}\right\}$ such that $0<\alpha_{i, \chi, \mu}<1$ and

$$
\sum_{i \in \mathbb{N}} \sum_{\chi \in \hat{F}} d_{\chi} \sum_{\mu=1}^{m_{i, \chi}} \alpha_{i, \chi, \mu}<+\infty .
$$

Suppose further that, for any $\chi \in \widehat{F}$, if there exists $i, i^{\prime} \in \mathbb{N}$ and $\mu=1, \ldots, m_{i, \chi}$, $\mu^{\prime}=1, \ldots, m_{i^{\prime}, \chi}$ such that $(i, \mu) \neq\left(i^{\prime}, \mu^{\prime}\right)$ but $w_{\delta_{\mu}}^{\chi}=w_{\delta_{\mu^{\prime}}}^{\chi}$ (where $\delta_{\mu} \in \Delta_{i, \chi}$ and $\left.\delta_{\mu^{\prime}} \in \Delta_{i, \chi}\right)$, then

$$
\mid\left(\operatorname{supp}(\varphi) \cap \alpha_{i, \chi, \mu}^{-1} \alpha_{i^{\prime}, \chi, \mu^{\prime}} \operatorname{supp}(\varphi) \mid=0 .\right.
$$

An explicit example is

$$
\begin{aligned}
\varphi & =\chi_{(1 / 2,1]}, \\
\alpha_{i, \chi, \mu} & =\frac{1}{2^{n_{i, \chi, \mu}}},
\end{aligned}
$$

where $(i, \chi, \mu) \mapsto n_{i, \chi, \mu}$ is any bijection from the index set

$$
\mathcal{N}=\left\{(i, \chi, \mu) \mid i \in \mathbb{N}, \chi \in \widehat{F}, m_{i, \chi}>0, \mu=1, \ldots, m_{i, \chi}\right\}
$$

onto $\mathbb{N}$.

With the above choices, define

$$
\varphi_{i, \chi, \mu}(\omega)=\varphi\left(\alpha_{i, \chi, \mu}^{-1} \omega\right) \quad \omega \in \widehat{\mathbb{R}}_{+} .
$$

Now, the sum in (3.25b contains products of the form

$$
\varphi\left(\frac{2^{j} \omega}{\alpha_{i, \chi, \mu}}\right) \varphi\left(\frac{2^{j} \omega}{\alpha_{i, \chi, \mu}}+\frac{2^{j} 2 \pi m}{\alpha_{i, \chi, \mu}}\right) .
$$

Since $\left|2^{j} 2 \pi m / \alpha_{i, \chi, \mu}\right|>\left|2^{j} 2 \pi m\right|>1$ for every odd integer $m$ and every non-negative integer $j$, one of the two factors must always vanish, so that $3.25 \mathrm{~b}$ holds true. Similarly, 3.31 implies 3.26a and 3.26b). 
3.4. Proof of Theorem 3.4. We first prove a technical lemma, which is a variant of a well known result (see Lemma 1.10 of [18]).

We recall that a family $\left(\psi_{i}\right)_{i \in \mathbb{N}}$ in a separable Hilbert space $\mathcal{H}$ is a Parseval frame if one of the following two equivalent conditions is satisfied:

a) for all $f \in \mathcal{H}$

$$
\sum_{i \in \mathbb{N}}\left\langle f, \psi_{i}\right\rangle \psi_{i}=f
$$

b) for all $f \in \mathcal{H}$

$$
\sum_{i \in \mathbb{N}}\left|\left\langle f, \psi_{i}\right\rangle\right|^{2}=\|f\|^{2},
$$

see Theorem 1.7 Chapter 7 of [18. Both series converge unconditionally. For a thorough discussion on frames see e.g. [23, 24].

Lemma 3.5. Let $\left(\psi_{i}\right)_{i \in \mathbb{N}}$ be a family of vectors in $\mathcal{H}$. If there exists a total subset $\mathcal{S}$ of $\mathcal{H}$ such that

a) for all $f \in \mathcal{S}$ the sequence $\left(\left\langle f, \psi_{i}\right\rangle\right)_{i \in \mathbb{N}}$ is in $\ell^{2}(\mathbb{N})$;

b) for all $f, g \in \mathcal{S}$

$$
\sum_{i \in \mathbb{N}}\left\langle f, \psi_{i}\right\rangle\left\langle\psi_{i}, g\right\rangle=\langle f, g\rangle
$$

then the family $\left(\psi_{i}\right)_{i \in \mathbb{N}}$ is a Parseval frame.

Proof. Define

$$
\mathcal{D}=\left\{\left.f \in \mathcal{H}\left|\sum_{i \in \mathbb{N}}\right|\left\langle f, \psi_{i}\right\rangle\right|^{2}<+\infty\right\}
$$

and $V: \mathcal{D} \rightarrow \ell^{2}(\mathbb{N})$

$$
V f=\left(\left\langle f, \psi_{i}\right\rangle\right)_{i \in \mathbb{N}} .
$$

By construction, $\mathcal{D}$ is a linear subspace containing $\mathcal{S}$, so that $\mathcal{D}$ is dense and $V$ is a linear operator. It is known that $V$ is a closed operator, see Proposition 2.8 of [5]. By (3.32), the restriction of $V$ to $\mathcal{S}$ preserves the scalar product. By linearity, the same property holds on the linear subspace spanned by $\mathcal{S}$, which is contained in $\mathcal{D}$ and dense in $\mathcal{H}$ since $\mathcal{S}$ is total in $\mathcal{H}$. Then $V$ extends to a unique isometry $W$ from $\mathcal{H}$ into $\ell^{2}(\mathbb{N})$. Since $V$ is closed, then $\mathcal{D}=\mathcal{H}$ and $V=W$. By definition of $V$, the family $\left(\psi_{i}\right)_{i \in \mathbb{N}}$ is a Parseval frame.

The following lemma is a variant of a result given in [5] in the context of admissible representations, see Proposition 2.23.

Lemma 3.6. Take two countable families $\left(\mathcal{H}_{j}\right)_{j \in \mathbb{N}}$ and $\left(\mathcal{H}_{j}^{\prime}\right)_{j \in \mathbb{N}}$ of separable Hilbert spaces, set $\mathcal{H}=\bigoplus_{j \in \mathbb{N}} \mathcal{H}_{j} \otimes \mathcal{H}_{j}^{\prime}$ and, for all $j \in \mathbb{N}$, denote the canonical projection by $P_{j}: \mathcal{H} \rightarrow \mathcal{H}_{j} \otimes \mathcal{H}_{j}^{\prime}$. A family $\left(\psi_{i}\right)_{i \in \mathbb{N}}$ is a Parseval frame for $\mathcal{H}$ if and only if the following two conditions hold true:

a) for all $j \in \mathbb{N}$ and all $f \in \mathcal{H}_{j}, f^{\prime} \in \mathcal{H}_{j}^{\prime}$

$$
\sum_{i \in \mathbb{N}}\left|\left\langle f \otimes f^{\prime}, P_{j} \psi_{i}\right\rangle\right|^{2}=\|f\|_{\mathcal{H}_{j}}^{2}\left\|f^{\prime}\right\|_{\mathcal{H}_{j}^{\prime}}^{2}
$$

b) for all $j, k \in \mathbb{N}, j \neq k$ and for all $f \in \mathcal{H}_{j}, f^{\prime} \in \mathcal{H}_{j}^{\prime}, g \in \mathcal{H}_{k}, g^{\prime} \in \mathcal{H}_{k}^{\prime}$

$$
\sum_{i \in \mathbb{N}}\left\langle f \otimes f^{\prime}, P_{j} \psi_{i}\right\rangle\left\langle P_{k} \psi_{i}, g \otimes g^{\prime}\right\rangle=0 .
$$


Proof. Assume that $\left(\psi_{i}\right)_{i \in \mathbb{N}}$ is a Parseval frame for $\mathcal{H}$ and fix $j \in \mathbb{N}$. Given $f \in \mathcal{H}_{j}$ and $f^{\prime} \in \mathcal{H}_{j}^{\prime}$, we have

$$
P_{j}^{*}\left(f \otimes f_{j}^{\prime}\right)=\sum_{i \in \mathbb{N}}\left\langle P_{j}^{*}\left(f \otimes f_{j}^{\prime}\right), \psi_{i}\right\rangle \psi_{i} .
$$

For all $k \in \mathbb{N}, P_{k}$ is a bounded linear operator, and $P_{k} P_{j}^{*}=\delta_{j k} P_{j} P_{j}^{*}=\delta_{j k} \operatorname{Id}_{\mathcal{H}_{j} \otimes \mathcal{H}_{j}^{\prime}}$. Then

$$
\begin{cases}\sum_{i \in \mathbb{N}}\left\langle f \otimes f_{j}^{\prime}, P_{j} \psi_{i}\right\rangle P_{j} \psi_{i}=f \otimes f_{j}^{\prime} & k=j \\ \sum_{i \in \mathbb{N}}\left\langle f \otimes f_{j}^{\prime}, P_{j} \psi_{i}\right\rangle P_{k} \psi_{i}=0 & k \neq j,\end{cases}
$$

whence a) and b) easily follow.

Conversely, set

$$
\mathcal{S}=\bigcup_{j \in \mathbb{N}}\left\{P_{j}^{*}\left(f \otimes f^{\prime}\right) \mid f \in \mathcal{H}_{j}, f^{\prime} \in \mathcal{H}_{j}^{\prime}\right\},
$$

which is total in $\mathcal{H}$ by construction. Conditions a) and b) imply that (3.32) of Lemma 3.5 is satisfied, hence, $\left(\psi_{i}\right)_{i \in \mathbb{N}}$ is a Parseval frame.

The following result is a restatement of the well known characterization of wavelet Parseval frames. For the sake of clarity, we set $\lambda=(j, k) \in \Lambda=\mathbb{Z}^{2}$ and $x_{\lambda}=$ $\left(2^{j} k, 2^{j}\right) \in \mathbb{R} \times \mathbb{R}_{+}$.

Lemma 3.7. If the family $\left\{\varphi_{i, \chi, \mu}\right\}$ in $L^{2}\left(\widehat{\mathbb{R}}_{+}\right)$satisfies (3.25a), 3.25b), (3.26a) and (3.26b), then

a) for each $i \in \mathbb{N}, \chi \in \widehat{F}$ and $\mu=1, \ldots, m_{i, \chi}$

$$
\sum_{\lambda \in \Lambda}\left|\left\langle\varphi, \widehat{W}^{+}\left(x_{\lambda}\right) \varphi_{i, \chi, \mu}\right\rangle_{2}\right|^{2}=\frac{1}{L}\|\varphi\|_{2}^{2}
$$

for all $\varphi \in L^{2}\left(\widehat{\mathbb{R}}_{+}\right)$;

b) for all $\chi \in \widehat{F}$, if there exists $i, i^{\prime} \in \mathbb{N}$ and $\mu=1, \ldots, m_{i, \chi}, \mu^{\prime}=1, \ldots, m_{i^{\prime}, \chi}$ such that $(i, \mu) \neq\left(i^{\prime}, \mu^{\prime}\right)$ but $w_{\delta_{\mu}}^{\chi}=w_{\delta_{\mu^{\prime}}}^{\chi}$ (where $\delta_{\mu} \in \Delta_{i, \chi}$ and $\left.\delta_{\mu^{\prime}} \in \Delta_{i, \chi}\right)$, then

$$
\sum_{\lambda \in \Lambda}\left\langle\varphi, \widehat{W}^{+}\left(x_{\lambda}\right) \varphi_{i, \chi, \mu}\right\rangle_{2}\left\langle\widehat{W}^{+}\left(x_{\lambda}\right) \varphi_{i^{\prime}, \chi, \mu^{\prime}}, \varphi^{\prime}\right\rangle_{2}=0
$$

for all $\varphi, \varphi^{\prime} \in L^{2}\left(\widehat{\mathbb{R}}_{+}\right)$.

Proof. The fact that (3.33) is equivalent to (3.25a and 3.25b is one of the fundamental results at the root of wavelet frames, see Theorem 1.6 of [18]. The fact that (3.26a) and (3.26b) imply (3.34) follows by Lemma 1.18 of [18, which, by polarization, can be rewritten as

$$
\begin{aligned}
& 2 \pi \sum_{\lambda \in \Lambda}\left\langle\varphi, \widehat{W}^{+}\left(x_{\lambda}\right) \varphi_{i, \chi, \mu}\right\rangle_{2}\left\langle\widehat{W}^{+}\left(x_{\lambda}\right) \varphi_{i^{\prime}, \chi, \mu^{\prime}}, \varphi^{\prime}\right\rangle_{2} \\
= & \int_{\widehat{\mathbb{R}}} \varphi(\omega) \overline{\varphi^{\prime}(\omega)} \sum_{j \in \mathbb{Z}} \varphi_{i^{\prime}, \chi, \mu^{\prime}}\left(2^{j} \omega\right) \overline{\varphi_{i, \chi, \mu}\left(2^{j} \omega\right)} \mathrm{d} \omega \\
+ & \int_{\widehat{\mathbb{R}}} \overline{\varphi^{\prime}(\omega)} \sum_{j \in \mathbb{Z}} \sum_{m \in 2 \mathbb{Z}+1} \varphi_{i^{\prime}, \chi, \mu^{\prime}}\left(\omega+2^{j} 2 \pi m\right) h_{m}\left(2^{j} \omega\right) \mathrm{d} \omega,
\end{aligned}
$$


where

$$
h_{m}(\omega)=\sum_{n=0}^{+\infty} \varphi_{i^{\prime}, \chi, \mu^{\prime}}\left(2^{n} \omega\right) \overline{\varphi_{i, \chi, \mu}\left(2^{n}(\omega+2 \pi m)\right)} .
$$

Indeed, 3.26a implies that the first summand vanishes, whereas 3.26b implies that $h_{m}$ vanish for all odd integers, hence the second summand is zero.

Proof of Theorem 3.4. By means of the unitary operator $S$, we can prove the result for the family of vectors

$$
S \pi\left(x_{\lambda, \ell}\right) \eta \quad \lambda \in \Lambda, \quad \ell=1, \ldots, L
$$

in the space $\bigoplus_{i \in \mathbb{N}} L^{2}\left(\widehat{\mathbb{R}}_{+}, \mathcal{H}_{i}\right)$, which by (3.11) and (3.21) can be identified with

$$
L^{2}\left(\widehat{\mathbb{R}}^{d}\right)=\bigoplus_{i \in \mathbb{N}, \chi \in \widehat{F}} \bigoplus_{\mu=1}^{m_{i, \chi}} L^{2}\left(\widehat{\mathbb{R}}_{+}, \mathcal{H}_{\chi} \otimes \mathbb{C}\left\{\epsilon_{\mu}\right\}\right)
$$

We will apply Lemma 3.6. So, let us fix $i, i^{\prime} \in \mathbb{N}, \chi, \chi^{\prime} \in \widehat{F}$ and $\mu \in\left\{1, \ldots, m_{i, \chi}\right\}$, $\mu^{\prime} \in\left\{1, \ldots, m_{i, \chi^{\prime}}\right\}$. Given $\varphi, \varphi^{\prime} \in L^{2}\left(\widehat{\mathbb{R}}_{+}\right)$and $w \in \mathcal{H}_{\chi}, w^{\prime} \in \mathcal{H}_{\chi^{\prime}}$, we look at the quantity

$$
\begin{aligned}
& A\left(i, \chi, \mu, i^{\prime}, \chi^{\prime}, \mu^{\prime}\right) \\
= & \sum_{\lambda \in \Lambda} \sum_{\ell=1}^{L}\left\langle\varphi \otimes w \otimes \epsilon_{\mu}, P_{i, \chi, \mu} S \pi\left(x_{\lambda, \ell}\right) \eta\right\rangle_{2}\left\langle P_{i^{\prime}, \chi^{\prime}, \mu^{\prime}} S \pi\left(x_{\lambda, \ell}\right) \eta, \varphi^{\prime} \otimes w^{\prime} \otimes \epsilon_{\mu^{\prime}}\right\rangle_{2} .
\end{aligned}
$$

Recall that, since $x_{\lambda, \ell}=\left(x_{\lambda}, R_{\ell}\right)$,

$$
P_{i, \chi, \mu} S \pi\left(x_{\lambda, \ell}\right) \eta=\widehat{W}^{+}\left(x_{\lambda}\right) \varphi_{i, \chi, \mu} \otimes \chi\left(R_{\ell}\right) w_{\delta_{\mu}}^{\chi} \otimes \epsilon_{\mu}
$$

hence we have

$$
\begin{aligned}
A\left(i, \chi, \mu, i^{\prime}, \chi^{\prime}, \mu^{\prime}\right) & =\left(\sum_{\lambda \in \Lambda}\left\langle\varphi, \widehat{W}^{+}\left(x_{\lambda}\right) \varphi_{i, \chi, \mu}\right\rangle_{2}\left\langle\widehat{W}^{+}\left(x_{\lambda}\right) \varphi_{i^{\prime}, \chi^{\prime}, \mu^{\prime}}, \varphi^{\prime}\right\rangle_{2}\right) \\
& \times\left(\sum_{\ell=1}^{L}\left\langle w, \chi\left(R_{\ell}\right) w_{\delta_{\mu}}^{\chi}\right\rangle_{\mathcal{H}_{\chi}}\left\langle\chi^{\prime}\left(R_{\ell}\right) w_{\delta_{\mu^{\prime}}}^{\chi^{\prime}}, w^{\prime}\right\rangle_{\mathcal{H}_{\chi^{\prime}}}\right),
\end{aligned}
$$

where the series are absolutely summable because of (3.33) and the Cauchy-Schwarz inequality.

From the Schur orthogonality relations applied to the pair of irreducible representations $\chi, \chi^{\prime}$ of $F$, we know that

$\frac{1}{L} \sum_{\ell=1}^{L}\left\langle w, \chi\left(R_{\ell}\right) w_{\delta_{\mu}}^{\chi}\right\rangle_{\mathcal{H}_{\chi}}\left\langle\chi^{\prime}\left(R_{\ell}\right) w_{\delta_{\mu^{\prime}}}^{\chi^{\prime}}, w^{\prime}\right\rangle_{\mathcal{H}_{\chi^{\prime}}}= \begin{cases}0 & \chi \neq \chi^{\prime} \\ \frac{1}{d_{\chi}}\left\langle w_{\delta_{\mu^{\prime}}}^{\chi}, w_{\delta_{\mu}}^{\chi}\right\rangle_{\mathcal{H}_{\chi}}\left\langle w, w^{\prime}\right\rangle_{\mathcal{H}_{\chi}} & \chi=\chi^{\prime} .\end{cases}$

Thus, if $\chi \neq \chi^{\prime}$, we get $A\left(i, \chi, \mu, i^{\prime}, \chi^{\prime}, \mu^{\prime}\right)=0$. From now on assume $\chi=\chi^{\prime}$, for which

$$
\begin{aligned}
A\left(i, \chi, \mu, i^{\prime}, \chi, \mu^{\prime}\right) & =L\left(\sum_{\lambda \in \Lambda}\left\langle\varphi, \widehat{W}^{+}\left(x_{\lambda}\right) \varphi_{i, \chi, \mu}\right\rangle_{2}\left\langle\widehat{W}^{+}\left(x_{\lambda}\right) \varphi_{i^{\prime}, \chi, \mu^{\prime}}, \varphi^{\prime}\right\rangle_{2}\right) \\
& \times \frac{1}{d_{\chi}}\left\langle w_{\delta_{\mu^{\prime}}}^{\chi}, w_{\delta_{\mu}}^{\chi}\right\rangle_{\mathcal{H}_{\chi}}\left\langle w, w^{\prime}\right\rangle_{\mathcal{H}_{\chi}} .
\end{aligned}
$$


If $(i, \mu) \neq\left(i^{\prime}, \mu^{\prime}\right)$ and $\delta_{\mu} \neq \delta_{\mu^{\prime}}$, then $A\left(i, \chi, \mu, i^{\prime}, \chi, \mu^{\prime}\right)=0$ since the family $w_{1}^{\chi}, \ldots, w_{d_{\chi}}^{\chi}$ is orthogonal. If $(i, \mu) \neq\left(i^{\prime}, \mu^{\prime}\right)$ but $\delta_{\mu}=\delta_{\mu^{\prime}}$, then by (3.34) it follows that $A\left(i, \chi, \mu, i^{\prime}, \chi, \mu^{\prime}\right)=0$. Finally, if $(i, \mu)=\left(i^{\prime}, \mu^{\prime}\right)$, then (3.22) yields

$$
\begin{aligned}
A(i, \chi, \mu, i, \chi, \mu) & =L\left(\sum_{\lambda \in \Lambda}\left\langle\varphi, \widehat{W}^{+}\left(x_{\lambda}\right) \varphi_{i, \chi, \mu}\right\rangle_{2}\left\langle\widehat{W}^{+}\left(x_{\lambda}\right) \varphi_{i, \chi, \mu}, \varphi^{\prime}\right\rangle_{2}\right)\left\langle w, w^{\prime}\right\rangle_{\mathcal{H}_{\chi}} \\
& =\left\langle\varphi, \varphi^{\prime}\right\rangle_{2}\left\langle w, w^{\prime}\right\rangle_{\mathcal{H}_{\chi}} \\
& =\left\langle\varphi \otimes w \otimes \epsilon_{\mu}, \varphi^{\prime} \otimes w^{\prime} \otimes \epsilon_{\mu}\right\rangle_{2},
\end{aligned}
$$

where the second equality is a consequence of (3.33).

Summarizing the above results in a single equation, we obtain

$$
A\left(i, \chi, \mu, i^{\prime}, \chi^{\prime}, \mu^{\prime}\right)= \begin{cases}\left\langle\varphi \otimes w \otimes \epsilon_{\mu}, \varphi^{\prime} \otimes w^{\prime} \otimes \epsilon_{\mu}\right\rangle_{2} & \text { if } \chi=\chi^{\prime} \text { and }(i, \mu)=\left(i^{\prime}, \mu^{\prime}\right), \\ 0 & \text { if } \chi \neq \chi^{\prime} \text { or }(i, \mu) \neq\left(i^{\prime}, \mu^{\prime}\right) .\end{cases}
$$

The conclusion follows from Lemma 3.6.

\section{ACKNOWLEDGMENTS}

G. S. Alberti was supported by the ERC Advanced Grant Project MULTIMOD267184. S. Dahlke was supported by Deutsche Forschungsgemeinschaft (DFG), Grant DA 360/19-1. F. De Mari and E. De Vito were partially supported by Progetto PRIN 2010-2011 "Varietà reali e complesse: geometria, topologia e analisi armonica". They are members of the Gruppo Nazionale per l'Analisi Matematica, la Probabilità e le loro Applicazioni (GNAMPA) of the Istituto Nazionale di Alta Matematica (INdAM).

The authors would like to thank Fulvio Ricci for suggesting the extension from the $2 \mathrm{D}$ case to the higher dimensional case.

\section{REFERENCES}

[1] G. S. Alberti, L. Balletti, F. De Mari, and E. De Vito. Reproducing subgroups of $S p(2, \mathbb{R})$. Part I: Algebraic classification. J. Fourier Anal. Appl., 19(4):651-682, 2013.

[2] G. S. Alberti, F. De Mari, E. De Vito, and L. Mantovani. Reproducing subgroups of $S p(2, \mathbb{R})$. Part II: admissible vectors. Monatsh. Math., 173(3):261-307, 2014.

[3] F. De Mari and E. De Vito. Admissible vectors for mock metaplectic representations. Appl. Comput. Harmon. Anal., 34(2):163-200, 2013.

[4] S. T. Ali, J.-P. Antoine, and J.-P. Gazeau. Coherent states, wavelets, and their generalizations. Theoretical and Mathematical Physics. Springer, New York, second edition, 2014.

[5] H. Führ. Abstract harmonic analysis of continuous wavelet transforms, volume 1863 of Lecture Notes in Mathematics. Springer-Verlag, Berlin, 2005.

[6] E. Cordero, F. De Mari, K. Nowak, and A. Tabacco. Analytic features of reproducing groups for the metaplectic representation. J. Fourier Anal. Appl., 12(2):157-180, 2006.

[7] E. Cordero, F. De Mari, K. Nowak, and A. Tabacco. Reproducing groups for the metaplectic representation. In Pseudo-differential operators and related topics, volume 164 of Oper. Theory Adv. Appl., pages 227-244. Birkhäuser, Basel, 2006.

[8] E. Cordero, F. De Mari, K. Nowak, and A. Tabacco. Dimensional upper bounds for admissible subgroups for the metaplectic representation. Math. Nachr., 283(7):982-993, 2010.

[9] E. Cordero and A. Tabacco. Triangular subgroups of $S p(d, \mathbb{R})$ and reproducing formulae. $J$. Funct. Anal., 264(9):2034-2058, 2013.

[10] E. J. King. Wavelet and frame theory:frame bound gaps, generalized shearlets, Grassmannian fusion frames, and p-adic wavelets. PhD thesis, University of Maryland, College Park, 2009.

[11] M. Duflo and C. C. Moore. On the regular representation of a nonunimodular locally compact group. J. Functional Analysis, 21(2):209-243, 1976. 
[12] H. G. Feichtinger and K. H. Gröchenig. Banach spaces related to integrable group representations and their atomic decompositions. I. J. Funct. Anal., 86(2):307-340, 1989.

[13] H. G. Feichtinger and K. H. Gröchenig. Banach spaces related to integrable group representations and their atomic decompositions. II. Monatsh. Math., 108(2-3):129-148, 1989.

[14] J. G. Christensen. Sampling in reproducing kernel Banach spaces on Lie groups. J. Approx. Theory, 164(1):179-203, 2012.

[15] J. G. Christensen and G. Ólafsson. Examples of coorbit spaces for dual pairs. Acta Appl. Math., 107(1-3):25-48, 2009.

[16] J. G. Christensen and G. Ólafsson. Coorbit spaces for dual pairs. Appl. Comput. Harmon. Anal., 31(2):303-324, 2011.

[17] H. Führ and K. Gröchenig. Sampling theorems on locally compact groups from oscillation estimates. Math. Z., 255(1):177-194, 2007.

[18] E. Hernández and G. Weiss. A first course on wavelets. Studies in Advanced Mathematics. CRC Press, Boca Raton, FL, 1996. With a foreword by Yves Meyer.

[19] S. Dahlke, F. De Mari, E. De Vito, D. Labate, G. Steidl, G. Teschke, and S. Vigogna. Coorbit spaces with voice in a Fréchet space. to appear on J. Fourier. Anal. Appl.

[20] I. Daubechies. Ten lectures on wavelets, volume 61 of CBMS-NSF Regional Conference Series in Applied Mathematics. Society for Industrial and Applied Mathematics (SIAM), Philadelphia, PA, 1992.

[21] J. L. Clerc. Analyse Harmonique, chapter "Les représentations des groupes compacts". C.I.M.P.A., 1981. Ecole "Analyse harmonique", 1980 Nancy (France).

[22] M. E. Taylor. Noncommutative harmonic analysis, volume 22 of Mathematical Surveys and Monographs. American Mathematical Society, Providence, RI, 1986.

[23] O. Christensen. An introduction to frames and Riesz bases. Applied and Numerical Harmonic Analysis. Birkhäuser Boston, Inc., Boston, MA, 2003.

[24] C. Heil. A basis theory primer. Applied and Numerical Harmonic Analysis. Birkhäuser/Springer, New York, expanded edition, 2011.

G. S. Alberti, Seminar for Applied Mathematics, Department of Mathematics, ETH Zürich, 8092 Zürich, Switzerland.

E-mail address: giovanni.alberti@math.ethz.ch

S. Dahlke, FB12 Mathematik und Informatik, Philipps-Universität Marburg, HansMeerwein Strasse, Lahnberge, 35032 Marburg, Germany.

E-mail address: dahlke@mathematik.uni-marburg.de

F. De Mari, Dipartimento di Matematica, Università di Genova, Via Dodecaneso 35, Genova, Italy.

E-mail address: demari@dima.unige.it

E. De Vito, Dipartimento di Matematica, Università di Genova, Via Dodecaneso 35, Genova, Italy.

E-mail address: devito@dima.unige.it

S. Vigogna, Department of Mathematics, Duke University, 120 Science Drive, 27708 Durham NC, United States.

E-mail address: stefano@math.duke.edu 